\title{
Determinação da concentração alveolar mínima do isofluorano em catetos (Tayassu tajacu) ${ }^{1}$
}

\begin{abstract}
Maria G.C. Oliveira ${ }^{2 *}$, Alessandro M.L. Morais², Amara G.A. Lima², Ariana L.C. Paiva $^{2}$, Talyta L. Nunes ${ }^{2}$, Yanna D.B. Passos ${ }^{2}$, Moacir F. Oliveira ${ }^{2}$ e Valéria V. Paula ${ }^{2}$

ABSTRACT.- Oliveira M.G.C., Morais A.M.L., Lima A.G.A., Paiva A.L.C., Nunes T.L., Passos Y.D.B., Oliveira M.F. \& Paula V.V. 2014. [Isoflurane minimum alveolar concentration in collared peccary (Tayassu tajacu).] Determinação da concentração alveolar mínima do isofluorano em catetos (Tayassu tajacu). Pesquisa Veterinária Brasileira 34(6):576-581. Departamento de Ciências Animais, Universidade Federal Rural do Semi-Árido, BR-110 Km 47, Presidente Costa e Silva, Mossoró, RN 59625-900, Brazil. E-mail: glauciacarlos@hotmail.com

Inhalation anesthesia has been widespread in veterinary medicine. Nevertheless, its use in wild animals is still limited, having no studies on its use been observed in the species. The objective of the research was to determine the isoflurane minimum alveolar concentration (MAC) in peccaries and present the effects of its administration on the hemodynamic and respiratory variables, as well as data concerning the anesthesia recovery. The study used 10 male animals with age ranging from one to three years, from the Centro de Multiplicação de Animais Silvestres of Universidade Federal Rural do Semi-Árido, Brazil. All the animals had anesthesia induced with propofol $7 \mathrm{mg} \cdot \mathrm{kg}^{-1}$, were intubated and connected to the anesthetic circuit with isoflurane and $100 \%$ oxygen. The supramaximal noxious stimulation used was the interdigital pinch, which was performed after 15 minutes of waiting for each provided isoflurane concentration. When negative response to the stimulus was observed, the concentration was reduced by $20 \%$; when positive response was verified, the stimulus was stopped, being the CAM value calculated from that point. Quantitative and qualitative data regarding recovery were also observed. Shapiro-Wilk test for normality was performed, as well as Levene test for equality of variance; the variables were subjected to One Way RM ANOVA for repeated measures followed by Tukey test, and the data expressed as mean and standard deviation. The MAC of isoflurane was $2.4 \%$ and the surgical MAC equal to $3.5 \%$. Depressive action of isoflurane on blood pressure, heart and breathing rates was observed when compared to the average of the variables on awake animals; however, during maintenance of anesthesia, they remained stable. Metabolic acidosis was observed in the pre-anesthetic, which was offset after inhalation anesthesia. Recovery was quiet and smooth. It was concluded that the isoflurane MAC for peccaries was greater than that observed in related species. Isoflurane can be used in this species, being considered safe and effective. The animals' recovery after anesthesia with isoflurane was free from excitement.
\end{abstract}

INDEX TERMS: Isoflurane, collared peccary, Tayassu tajacu, anesthesia, wild animal, supramaximal stimulation, recovery.

RESUMO.- A anestesia inalatória vem sendo amplamente difundida na medicina veterinária, no entanto seu uso em animais selvagens ainda é restrito, não sendo observado

\footnotetext{
${ }^{1}$ Recebido em 6 de fevereiro de 2014.

Aceito para publicação em 29 de abril de 2014.

${ }^{2}$ Departamento de Ciências Animais, Universidade Federal Rural do Semi-Árido (Ufersa), BR-110 Km 47, Cx. Postal 137, Presidente Costa e Silva, Mossoró, RN 59625-900, Brasil.*Autor para correspondência: glauciacarlos@ hotmail.com
}

nenhum estudo referente à sua utilização na espécie Tayassu tajacu. 0 objetivo da pesquisa foi determinar a concentração alveolar mínima (CAM) do isofluorano em catetos e apresentar os efeitos desta administração sobre as variáveis hemodinâmicas e respiratórias, como também a qualidade da recuperação anestésica. Utilizou-se 10 animais, machos, com idade variando de 1 a 3 anos oriundos do Centro de Multiplicação de Animais Silvestres da Universidade Federal Rural do Semi-Árido, Brasil. Todos os animais 
tiveram anestesia induzida com $7 \mathrm{mg} \cdot \mathrm{kg}^{-1}$ de propofol e posteriormente foram conectados a circuito anestésico com isofluorano e oxigênio $100 \%$. 0 estímulo noceptivo supramáximo adotado foi pinçamento interdigital, o qual era realizado após 15 minutos de espera para cada concentração de isofluorano fornecida. Ao ser observada resposta negativa frente ao estímulo a concentração era reduzida em $20 \%$, quando verificada resposta positiva o estímulo era cessado, calculando-se a partir daí o valor da CAM. Observou-se dados quantitativos e qualitativos referentes à recuperação. Utilizou-se o teste de normalidade de Shapiro Wilk e de homogeneidade de variânica de Levene, as variáveis avaliadas foram submetidas à One Way ANOVA-RM para medidas repetidas, seguidas por Teste Tukey, sendo os dados expressos em média e desvio padrão. A CAM do isofluorano foi de 2,4\%, sendo a CAM cirúrgica igual a 3,5\%. Observou-se ação depressiva do isofluorano sobre a pressão arterial, frequência cardíaca e respiratória quando comparada a média dessas variáveis para animais acordados, entretanto durante a manutenção anestésica mantiveram-se estáveis. Observou-se acidose metabólica no período pré-anestésico o qual foi compensado após a realização da anestesia inalatória. A recuperação anestésica foi tranquila e rápida. Concluiu-se que a CAM do isofluorano para catetos foi maior que a observada em espécies afins. 0 isofluorano pode ser utilizado nesta espécie, sendo considerado seguro e eficaz. A recuperação dos animais após anestesia com isofluorano foi livre de excitação.

TERMOS DE INDEXAÇÃO: Isofluorano, catetos, Tayassu tajacu, anestesia, animal selvagem, estímulo supramáximo, recuperação.

\section{INTRODUÇÃO}

A captura e contenção química de animais selvagens geralmente são conduzidas sob circunstâncias difíceis. 0 acesso e melhoria de tais procedimentos são partes importantes na conservação e manejo de animais de vida livre e em cativeiro (Fahlman 2005). 0 uso de doses excessivas ou uma analgesia inadequada são exemplos de situações que ocorrem por falta de um padrão de referência para a espécie selvagem em estudo e acabam interferindo na indução e recuperação anestésica (Farias et al. 2009).

Os catetos (Tayassu tajacu) são animais gregários e rústicos que produzem carne e couro de excelente qualidade (Costa et al. 2004). Batista et al. (2008) destacam esses animais dentre as espécies silvestres da fauna brasileira que vem demonstrando condições favoráveis à adaptação em cativeiro e consequente exploração comercial.

O isofluorano é um anestésico inalatório amplamente empregado em medicina veterinária devido ao seu baixo coeficiente de solubilidade sangue/gás, o que permite alterações rápidas no plano anestésico e um período de recuperação anestésica relativamente curto (Eger 2001). Uma das maneiras de se avaliar a potência de determinado agente inalatório é por meio da concentração alveolar mínima (CAM). Na anestesia, um método de determinação objetiva da CAM é de fundamental importância, já que os agentes de anestesia inalatória causam depressão do sistema nervoso central (SNC) e cardiorrespiratório (Steffey \& Mama 2013).
Visto a importância dos anestésicos inalatórios na anestesiologia veterinária atual e a escassez de relatos sobre o uso de tais agentes na espécie supracitada, o objetivo desse estudo foi determinar a CAM do isofluorano em catetos. Bem como avaliar as variáveis cadiorrespiratórias e a recuperação anestésica e desta forma assegurar ou não a utilização dos agentes voláteis de anestesia na espécie.

\section{MATERIAL E MÉTODOS}

O estudo foi submetido e aprovado pela Comissão de Ética no Uso de Animais (CEUA) da Universidade Federal Rural do Semi-Árido (UFERSA), protocolo 23091.000530/2012-23. E está registrado junto ao Sisbio - IBAMA, sob número 32085-1.

Foram utilizados 10 catetos (Tayassu tajacu), machos, pesando em média $20 \mathrm{~kg}$ e idade variando de 1 a 3 anos, oriundos do Centro de Multiplicação de Animais Silvestres da UFERSA, situado no nordeste do Brasil (Mossoró, RN, Brasil; 5¹0, 37²1' W). Os animais foram submetidos a prévio jejum sólido e hídrico de 12 e 6 horas, respectivamente. 0 procedimento foi realizado sempre no mesmo horário (05:00h às 11:00h da manhã) a fim de se evitar alterações nos valores da CAM que pudessem sofrer interferência do rítmo circadiano.

A indução da anestesia foi realizada através da administração intravenosa de $7 \mathrm{mg} . \mathrm{kg}^{-1}$ de propofol, a partir daí foi realizada infusão $10 \mathrm{~mL} / \mathrm{kg} / \mathrm{h}$ de solução de Rínger com lactato de sódio. A intubação foi procedida logo em seguida com auxílio de um guia rígido e iluminado na sua porção distal. Em seguida, os animais foram conectados a circuito com absorvedor de $\mathrm{CO}_{2}$ com oxigênio a $100 \%$ em fluxo de $1,5 \mathrm{~L} / \mathrm{min}$ associado ao isofluorano. Foi realizada ventilação assistida, adotando-se $\mathrm{ETCO}_{2}$ (concentração expirada de dióxido de carbono) entre 35-40mmHg. Acoplou-se um sensor à extremidade distal da sonda endotraqueal para obtenção dos valores de $f$ (frequência respiratória), ETCO ${ }_{2}$, Fliso (concentração inspirada de isofluorano) e ETiso (concentração expirada de isofluorano), sendo monitoradas continuamente através de analisador de gases. Fixou-se um cateter na artéria dorsal podal para determinação dos valores de pressão arterial sistêmica invasiva, a qual juntamente com a frequência e ritmo cardíaco foram monitorados por meio de monitor multiparamétrico. A pressão arterial média (PAM) foi mantida em $60 \mathrm{mmHg}$ através da infusão de efedrina $10 \mu \mathrm{g} / \mathrm{kg} / \mathrm{min}$ e somente foi utilizada quando esta variável encontrava-se abaixo do valor referido e durante observação de respostas negativas frente ao estímulo, ou seja, após constatação da única resposta positiva a infusão de efedrina era cessada, para assim serem observados somente os efeitos do isofluorano sobre os parâmetros avaliados. A T (temperatura corporal) foi mensurada através de um sensor de temperatura cuja extremidade distal foi posicionada na região do esôfago torácico, sendo mantida entre 37 e $38^{\circ} \mathrm{C}$ com auxílio de um colchão térmico. Todas as variáveis foram avaliadas continuamente durante todo transcorrer do experimento, entretanto para fins de análise foram adotados quatro momentos específicos: valores basais; após os animais estarem sob anestesia inalatória a uma concentração de 3\% de isofluorano; após a determinação da 1 CAM e por fim, ao fornecimento da concentração correspondente à 1,5 CAM. Os valores de hemogasometria arterial foram analisados através de analisador portátil e avaliados nos momentos supracitados.

A CAM do isofluorano foi determinada similarmente ao descrito por Quasha et al. (1980) e iniciou-se após passados $30 \mathrm{mi}$ nutos da última administração de propofol. A ETiso inicial de 3\% determinada em estudo piloto Cada ETiso utilizada foi mantida estável por um período de 15 minutos antes da realização do es- 
tímulo doloroso, o qual compreendia o pinçamento interdigital com pinça hemostática fechada até a primeira cremalheira, durante um minuto, ou menos, caso fosse observado uma resposta positiva. Ao término do estímulo, caso não fosse obtida resposta positiva, a ETiso era então reduzida em $20 \%$, sendo este procedimento repetido até que o animal apresentasse resposta positiva, assim que esta era obtida, o estímulo era cessado imediatamente. A CAM era então calculada a partir da média aritmética da mais alta concentração que permitia uma resposta motora e a mais baixa concentração que previnia uma resposta positiva. As respostas positivas compreendiam qualquer ação realizada pelo animal durante o minuto do pinçamento, podendo ser movimento de cabeça, flexão do membro estimulado ou algum outro e deglutição, e era considerada negativa quando o indivíduo permanecia imóvel e indiferente. A partir do valor da CAM foi calculado também a CAM cirúgica (1,5 x CAM) e essa concentração foi fornecida ao animal. Após essa etapa, o fornecimento do isofluorano era interrompido e marcava-se a partir daí o período de recuperação anestésica, determinados em tempo de extubação, caracterizado pela volta do reflexo laringotraqueal; primeiro movimento de cabeça; posição em decúbito esternal; posição quadrupedal e por fim o caminhar. Avaliou-se ainda a recuperação de forma qualitativa através de alternativa binária, onde o escore 0 (zero) correspondeu a recuperação ruim, escore 1 (um) a recuperação boa.

Todos os dados foram avaliados quanto à normalidade pelo teste de Shapiro-Wilk e homogeneidade por Levene. Os parâmetros fisiológicos e hemogasométricos foram submetidos à análise de variância (One way ANOVA RM) seguidas por Teste de Tukey. Valores de $\mathrm{P}<0,05$ foram considerados significativos.

\section{RESULTADOS}

A CAM do isofluorano para catetos foi de 2,35 $20,24 \%$, os valores médios, máximos e mínimos, assim como os valores de 1,5 CAM e a CAM em que os animais apresentaram os primeiros sinais de extubação estão expressos no Quadro 1. Durante todo o experimento não foram obervadas intercorrências devido à administração de isofluorano aos animais. 0 período para determinação da CAM foi em média $5 \pm 0,4$ horas.

Com relação aos parâmetros vitais analisados, apenas a T corporal não sofreu influência da anestesia com isofluorano. As demais variáveis apresentaram depressão significativa quando comparadas às médias para animais acordados, contudo durante a manutenção anetésica permaneceram iguais, com excessão da frequência cardíaca (FC) e pressão arterial diastólica (PAD) que diminuíram significativamente durante o fornecimento de 1,5 CAM. A hemogasometria constatou que os animais encontravam-se de acidose metabólica antes da anestesia. Ao serem anestesiados, o distúrbio foi compensado e os valores de pH mostraram-se dentro do intervalo considerado normal (Quadro 2).

No que concerne à avaliação qualitativa da recuperação anestésica, observamos que a maioria dos animais (80\%) apresentaram poucas tentativas de levantar-se e que esse momento foi livre de agitação, demonstrando recuperação do tipo gradativa. Apenas 20\% mostraram sinais violentos de recuperação, caracterizados por tentativas desastradas e

Quadro 1. Valores de CAM do isofluorano em catetos (Tayassu tajacu) submetidos ao pinçamento interdigital

\begin{tabular}{lcccc}
\hline Variáveis (\%) & Média \pm DP & Mín. & Máx. & CV \\
\hline 1 CAM & $2,35 \pm 0,24$ & 2,2 & 2,7 & 10,2 \\
1,5 CAM & $3,54 \pm 0,38$ & 3,3 & 4,1 & 10,9 \\
CAM da extubação & $0,67 \pm 0,23$ & 0,3 & 1,0 & 35,2
\end{tabular}

$\overline{\mathrm{CAM}}$ = concentração alveolar mínima; DP = desvio padrão; Mín = mínimo; Máx = máximo; $\mathrm{CV}=$ coeficiente de variação.

Quadro 2. Valores médios e desvio padrão das funções vitais e hemogasométricas de catetos (Tayassu tajacu) anestesiados com isofluorano e submetidos ao pinçamento interdigital, em momentos diferentes (animais sem anestesia, concentração inicial de $3 \%$ de isofluorano, 1 CAM e 1,5 CAM)

\begin{tabular}{lcccc}
\hline Variáveis & Animais sem anestesia & $3 \%$ de isofluorano & 1 CAM & 1,5 CAM \\
\hline FC (bmp) & $129,7 \pm 13,79^{\mathrm{a}}$ & $100,2 \pm 9,98^{\mathrm{bc}}$ & $101,90 \pm 1,10^{\mathrm{ab}}$ & $95,80 \pm 12,73^{\mathrm{c}}$ \\
f (mpm) & $44,80 \pm 9,39^{\mathrm{a}}$ & $14,80 \pm 5,97^{\mathrm{b}}$ & $20,00 \pm 10,23^{\mathrm{b}}$ & $19,60 \pm 8,07^{\mathrm{b}}$ \\
T ( $\left.{ }^{\circ} \mathrm{C}\right)$ & $37,40 \pm 0,97^{\mathrm{a}}$ & $37,70 \pm 0,47^{\mathrm{a}}$ & $38,03 \pm 0,14^{\mathrm{a}}$ & $38,00 \pm 0,34^{\mathrm{a}}$ \\
PAS (mmHg) & $171,1 \pm 27,76^{\mathrm{a}}$ & $90,20 \pm 34,38^{\mathrm{b}}$ & $95,20 \pm 29,42^{\mathrm{b}}$ & $84,33 \pm 29,24^{\mathrm{b}}$ \\
PAD (mmHg) & $91,0 \pm 30,35^{\mathrm{a}}$ & $46,80 \pm 6,35^{\mathrm{b}}$ & $42,00 \pm 13,93^{\mathrm{bc}}$ & $33,88 \pm 12,79^{\mathrm{c}}$ \\
PAM (mmHg) & $113,0 \pm 33,35^{\mathrm{a}}$ & $65,3 \pm 7,66^{\mathrm{b}}$ & $63,30 \pm 20,10^{\mathrm{b}}$ & $51,55 \pm 17,30^{\mathrm{b}}$ \\
pH & $7,15 \pm 0,07^{\mathrm{b}}$ & $7,41 \pm 0,04^{\mathrm{a}}$ & $7,41 \pm 0,03^{\mathrm{a}}$ & $7,44 \pm 0,03^{\mathrm{a}}$ \\
PaCO2 (mmHg) & $40,0 \pm 6,58^{\mathrm{a}}$ & $42,40 \pm 4,19^{\mathrm{a}}$ & $43,41 \pm 4,18^{\mathrm{a}}$ & $46,05 \pm 3,49^{\mathrm{a}}$ \\
PaO2 (mmHg) & $72,7 \pm 10,1^{\mathrm{c}}$ & $361,88 \pm 113,45^{\mathrm{b}}$ & $406,66 \pm 108,95^{\mathrm{ab}}$ & $370,0 \pm 75,64^{\mathrm{a}}$ \\
Beef (mmol/L) & $-14,50 \pm 3,06^{\mathrm{c}}$ & $2,90 \pm 3,84^{\mathrm{b}}$ & $5,40 \pm 2,27^{\mathrm{a}}$ & $5,50 \pm 1,84^{\mathrm{a}}$ \\
HCO3 (mmol/L) & $14,08 \pm 2,47^{\mathrm{c}}$ & $27,18 \pm 3,37^{\mathrm{b}}$ & $29,36 \pm 2,20^{\mathrm{ab}}$ & $29,52 \pm 1,63^{\mathrm{a}}$ \\
TCO2 (mmol/L) & $15,2 \pm 2,61^{\mathrm{b}}$ & $28,30 \pm 3,52^{\mathrm{a}}$ & $30,60 \pm 2,41^{\mathrm{a}}$ & $30,70 \pm 1,63^{\mathrm{a}}$ \\
sO2 (\%) & $87,6 \pm 4,60^{\mathrm{b}}$ & $99,40 \pm 1,57^{\mathrm{a}}$ & $99,00 \pm 3,16^{\mathrm{a}}$ & $100,0^{\mathrm{a}}$ \\
Hct (\%) & $51,5 \pm 3,89^{\mathrm{a}}$ & $37,30 \pm 5,65^{\mathrm{b}}$ & $36,50 \pm 4,94^{\mathrm{b}}$ & $35,90 \pm 4,95^{\mathrm{b}}$ \\
Hb (g/dL) & $17,51 \pm 1,33^{\mathrm{a}}$ & $12,68 \pm 1,92^{\mathrm{b}}$ & $12,40 \pm 1,69^{\mathrm{b}}$ & $12,22 \pm 1,66^{\mathrm{b}}$
\end{tabular}

$\overline{\mathrm{FC}}$ = frequência cardíaca; $\mathrm{bpm}=$ batimentos por minuto; $\mathrm{f}=$ frequência respiratória; $\mathrm{mpm}=\mathrm{movi}$ mentos por minuto; $\mathrm{T}$ = temperatura; $\mathrm{T}$ = temperatura; $\mathrm{PAS}=$ pressão arterial sistólica; $\mathrm{mmHg}$ = milímetros de mercúrio; PAD = pressão arterial distólica; $\mathrm{PAM}=$ pressão arterial média; $\mathrm{pH}=$ potencial hidrogeniônico; PCO2 = pressão parcial de dióxido de carbono; $\mathrm{mmHg}$ = milímetros de mercúrio; PO2 = pressão parcial de oxigênio; Beef =: excesso de base; HCO3 = bicarbonato de sódio; $\mathrm{mmol} / \mathrm{L}=$ : milimol por litro; TCO2 = concentração sérica total de dióxido de carbono; sO2 = saturação da oxi-hemoglobina; \% = porcentagem; Hct = hematócrito; Hb = hemoglobina; g/dL = gramas por decilitro. $\mathrm{a}, \mathrm{b}, \mathrm{c}$ Letras diferentes na linha significam diferença estatística $(\mathrm{P}<0,05)$. 
repetidas de levantar-se, associado à incoordenação quando da posição quadrupedal. No Quadro 3 estão expostos os valores quantitativos referentes à recuperação anestésica. Pode-se afirmar que o tempo médio decorrido desde que o animal ficou em decúbito esternal até o início da caminhada foi relativamente curto, conotando despertar tranquilo.

\section{Quadro 3. Valores referentes à recuperação anestésica de catetos (Tayassu tajacu) anestesiados com isofluorano submetidos ao pinçamento interdigital}

\begin{tabular}{lcccc}
\hline Tempos (min) & Média \pm DP & Mín. & Máx. & CV (\%) \\
\hline Extubação & $18,9 \pm 12,09$ & 6,0 & 43,0 & 64,0 \\
Movimento de cabeça & $32,1 \pm 15,19$ & 14,0 & 64,0 & 47,3 \\
Decúbito esternal & $41,0 \pm 18,71$ & 21,0 & 85,0 & 45,6 \\
Posição quadrupedal & $43,3 \pm 18,8$ & 24,0 & 89,0 & 43,5 \\
Caminhar & $43,5 \pm 18,62$ & 26,0 & 89,0 & 42,8
\end{tabular}

Min = minutos; $\mathrm{DP}$ = desvio padrão; Mín = mínimo; Máx = máximo; $\mathrm{CV}=$ coeficiente de variação.

\section{DISCUSSÃO}

Esse estudo é pioneiro no que diz respeito ao uso de anestésicos inalatórios em catetos. Foi escolhido o isofluorano devido ser o fármaco atualmente mais empregado em medicina humana e veterinária sendo o mais indicado em casos de pacientes de risco e ainda por apresentar a melhor relação custo benefício. A CAM de 2,35 $\pm 0,24 \%$ encontrada está acima da CAM média encontrada para suínos por Haga, Ranheim \& Spadavecchia (2011) que foi de 1,9\%. Dois fatores contribuíram para disparidade observada: a espécie diferente e o estímulo aplicado, o qual no último estudo foi empregado fórceps na região caudal numa área compreendendo $1 \mathrm{~cm}^{2}$. 0 pinçamento do dedo vestigial foi o estímulo empregado em outro estudo em suínos, que relevou valor de CAM do isofluorano de 2,05 $\pm 0,28 \%$ (Kurita et al. 2007). Distintamente dessas duas pesquisas, Greene et al. (2004) apresentaram como sendo $1,3 \pm 0,2 \%$ a CAM do isofluorano para suínos submetidos ao pinçamento na base da cauda. De acordo com relatos de Oliva \& Fantoni (2009), a CAM do isofluorano para as espécies domésticas fica em torno de 1,4\%, o que não se aplica aos catetos, dado serem animais selvagens.

A partir do momento em que se padroniza a estimulação nociceptiva supramáxima que será utilizada em determinado estudo, a caracterização da resposta motora frente ao estímulo se torna a principal responsável pela variabilidade nos valores de CAM. A maioria dos relatos encontrados na literatura (Steffey \& Howland 1978, Bloor \& Flacke 1982, Valverde et al. 2003) se baseia na descrição padrão de Eger et al. (1965) para classificar a resposta motora frente à estimulação nociceptiva, em positiva ou negativa. A classificação proposta por Eger et al. (1965) se mostrou eficiente para uso em catetos, o que foi confirmado antes do início do estudo através da realização do projeto piloto.

Foi imprescindível o uso de agente injetável indutor da anestesia, de rápida latência e período de ação, visto a espécie em estudo ser extremamente agressiva, os dentes caninos são protuberantes e ficam para fora da boca, impossibilitando por questões de segurança, o uso de másca- ra para indução com isofluorano. Além disso, tem-se o fato consolidado de que o isofluorano em humanos e nos mamíferos em geral possui odor pungente e promove irritação de mucosas, o que não o torna o agente mais indicado para indução da anestesia sem prévia aplicação de medicação pré-anestésica (Doi \& Ikeda 1978). Desta forma, foi escolhido a utilização do propofol para indução da anestesia por ser um fármaco que, embora possua ampla distribuição pela sua característica lipofílica, é um agente que desaparece rapidamente do plasma devido a propriedade de metabolização hepática e extra-hepática (Langley \& Heel 1988). Estudos farmacocinéticos utilizando um modelo bicompartimental demonstraram uma meia-vida de distribuição inicial de 2 a 8 minutos que é responsável pelo término do efeito hipnótico, e uma meia vida de eliminação de 1 a 3 horas (Oliveira \& Elias 2006). Devido a estas características farmacocinéticas do propofol, a meia hora de espera entre a aplicação do bolus de indução do propofol e início da deteminação da CAM, acrescidos aos quinze minutos de equilíbrio para cada valor de CAM do isofluorano a ser pesquisado no animal, acreditamos que provavelmente a dose do bolus de indução do propofol não tenha influenciado na determinação da CAM. A escolha deste agente também se deu ao fato do propofol já ter sido empegado em catetos demonstrando eficácia na indução anestésica (Souza et al. 2009).

Preliminarmente, utilizou a uma concentração de 1,9\% proposta por Haga et al. (2011) para suínos, entretanto foi testemunhado os catetos apresentando sinais clássicos de superficialização da anestesia, além de resposta positiva frente ao primeiro estímulo supramáximo aplicado. Desta forma, resolveu-se elevar a concentração inicial para 4\%, nesse novo valor os animais mostraram pronunciada hipotensão, sendo então o valor ajustado gradualmente até chegarmos a concentração expirada de $3 \%$ onde os animais ainda respondiam negativamente frente ao estímulo supramáximo, sendo assim esse valor empregado como concentração inicial padrão para todos os animais.

Os valores encontrados para FC aproximam-se dos mostrados por Greene et al. (2004) ao determinarem a CAM do isofluorano para suínos, entretanto quando fornecida 1,5 CAM nesse mesmo estudo não foi testemunhado discrepância de valores, fato contrário ao da na nossa pesquisa, na qual foram significativamente mais baixos com 1,5 CAM. Embora seja sabido que o isofluorano cause taquicardia de forma compensatória a fim de manter o débito cardíaco devido sua ação periférica vasodilatadora dose-dependente (Golden et al. 1998) este fato não foi observado nos resultados. Contrariamente, o que se viu foi redução gradativa na FC, onde os animais mostraram certa bradicardia ao final do experimento.

Mesmo inciando com 3\% de isofluorano, os animais continuaram a apresentar hipotensão, sendo necessária utilização de fármacos vasoativos. A hipotensão é considerada um dos fatores que podem alterar a CAM, diminuindo o requerimento dos anestésicos inalatórios (Quasha et al. 1980). A dobutamina é o agente vasoativo utilizado para tratar a hipotensão durante a anestesia em cavalos (Fantoni et al. 2013). Este agente $\beta-1$ agonista melhora a 
contratilidade miocárdica, a qual é a principal causa da hipotensão causada pelo halotano (Hellyer et al. 1991). Em animais anestesiados pelo isofluorano, entretanto, tem sido sugerido que a contratilidade miocárdica é preservada quando comparado ao halotano e que a redução da resistência vascular sistêmica é a principal causa de hipotensão em animais anestesiados com isofluorano (Raisis et al. 2000). Por isso, drogas vasoativas de ação $\alpha$-adrenérgicas tem sido apontadas como de eleição para uso durante anestesia com esse halogenado (Egger et al. 2009). A efedrina foi usada a fim de manter a PAM no valor mínimo de 60 $\mathrm{mmHg}$, contudo somente foi empregada até obtenção da única resposta positiva frente ao estímulo. 0 uso da efedrina pode ser considerado uma limitação deste estudo, pois a efedrina é uma substância sintética, não-catecolamina, simpatomimética que atua direta e indiretamente (através da liberação de norepinefrina das terminações nervosas simpáticas) nos receptores alfa e beta adrenérgicos (Egger et al. 1999). A PAM durante o fornecimento de 1 CAM foi de $63,30 \pm 20,10 \mathrm{mmHg}$ e manteve-se igual durante todo experimento, diferindo apenas dos valores basais. Os nossos resultados assemelham-se à média de $66 \mathrm{mmHg}$ para suínos anestesiados com isofluorano (Greene et al. 2004), porém mostraram-se acima dos resultados de Lundeen, Manohar \& Parks (1983) que encontraram 87 e $69 \mathrm{mmHg}$ ao determinarem a 1 CAM e 1,5 CAM do isofluorano para suínos, respectivamente.

A taquipnéia em que se encontrava os animais no primeiro contato denota o efeito da conteção física necessária para realização de trabalhos em animais selvagens. A $f$ foi mantida de forma assistida através de ventilação manual, sempre a fim de manter a ETCO $_{2}$ entre $35-45 \mathrm{mmHg}$ (Haskins 2013). Essa variável diminuiu significativamente após indução anestésica e permaneceu inalterada durante a manutenção da anestesia. É conhecido o efeito depressor dose-dependente do isofluorano sobre a $f$, conforme relatos de Galloway et al. (2003), o isofluorano promoveu redução mais pronunciada na $f$ quando comparado ao sevoflurano, em concentrações crescentes, podendo ocorrer ainda diminuição do volume-minuto e elevação da $\mathrm{PaCO}_{2}$, contudo quadros de hipercapnia não foram vistos no nosso estudo. Podemos afirmar, portanto que a anestesia com isofluorano promoveu melhor qualidade de respiração nos animais, principalmente quando comparamos às variáveis respiratórias de catetos anestesiados com cetamina/diazepam (Souza et al. 2008) e de javalis tratados com tiletamina/zolazepam (Sutherland-Smith et al. 2004).

0 desequilíbrio ácido básico envolvendo uma situação de acidose metabólica, visto nos catetos não anestesiados da pesquisa em tela, provavelmente está associada a ativação da primeira resposta dos diferentes mecanismos de tamponamento do organismo: os tampões químicos, que atuam em segundos para resistir ou minimizar as alterações na concentração de íons $\mathrm{H}^{+}$e representam a linha inicial de defesa do organismo contra alterações no pH (Muir \& Morais 2013). Porém, esse quadro não foi observado por Souza et al. (2008) no qual os animais apresentaram estado de acidose respiratória, sendo atribuída pelo autor ao estresse desencadeado com a contenção dos animais. Sabe- -se que durante o jejum prolongado, existe uma tendência de ocorrer acidose metabólica leve pelo acúmulo de corpos cetônicos e pela formação de ácidos graxos livres (Ortolani 2003). Desta forma, esse fato pode também ter contribuído para a o quadro instalado. De acordo com Dahl, Woldseth \& Lindemann (2012) o jejum leva a diminuição na liberação de insulina, a lipólise e a produção de corpos cetônicos, e essa produção é exacerbada com a presença de estresse e desse modo agrava o quadro de acidose metabólica. Após serem anestesiados, e desta forma aumentar a oferta de oxigênio, a qual passou de uma $\mathrm{PaO} 2$ inicial de $72,7 \pm 10$ para $361,88 \pm 113,45 \mathrm{mmHg}$, a situação de acidose metabólica desapareceu e os animais passaram a apresentar $\mathrm{pH}$ dentro de valores normais.

Foi observada redução significativa nos valores de $\mathrm{Ht}$ e Hb após a anestesia, estando esse fato associado a utilização de propofol que promove sequestro de hemácias para sítios não esplênicos após sua utilização (Wilson et al. 2004). Durante a manutenção da anestesia com isofluorano os valores de $\mathrm{Ht}$ e $\mathrm{Hb}$ permaneceram iguais, o mesmo aconteceu com suínos anestesiados com isofluorano e sevofluorano (Kimme et al. 2007).

A maioria dos animais recuperou-se de forma tranquila e gradativa, sem que fosse observada agitação ou sinais de canibalismo, conforme relatado comum na espécie suína (Pulz 2006). Os primeiros sinais de recuperação consistiram em movimento de cabeça, seguidos de decúbito esternal, depois posição quadrupedal onde seguiam rapidamente para junto do grupo, possivelmente assustados com a presença do observador. Contudo, dois animais mostraram-se agitados, apresentando tentativas repetidas de levantar-se sem sucesso e ainda incoordenação e queda ao caminharem. Esse fato pode estar associado à variação individual e também a não utilização de medicação pré-anestésica, já que essa favorece uma indução suave e assegura recuperação de maneira tranquila e calma (Lukasik 1999). Relatos de Pulz (2006) afirmam que $100 \%$ dos suínos que tiveram anestesia mantida somente com isofluorano apresentaram recuperação tranquila e sem sinais de excitação, diferente do que ocorreu quando a manutenção foi realizada com isofluorano, fentanil e ainda com o uso de bloqueadores neuromusculares, onde o autor enaltece sinais de excitação durante esse período, sendo o fentanil julgado como o responsável por uma recuperação laboriosa. De modo geral, verifica-se que há certa carência de informações acerca do uso de outros protocolos de anestesia geral, além da associação tiletamina-zolazepam ou cetamina-diazepam, para a catetos.

\section{CONCLUSÕES}

Concluiu-se desse modo que a anestesia inalatória com isofluorano mostrou-se segura e eficaz para a espécie.

A recuperação dos animais após anestesia com administração de isofluorano foi livre de excitação.

Agradecimentos.- Ao CNPq no55237/2011-5 pelo apoio financeiro. À Coordenação de Aperfeiçoamento de Pessoal de Nível Superior (CAPES), pela concessão de bolsa de mestrado e ao CEMAS-UFERSA pela disponibilidade. 


\section{REFERÊNCIAS}

Batista J.S., Bezerra F.S.B., Lira R.A., Orpinelli S.R.T., Dias C.E.V. \& Oliveira A.F. 2008. Síndrome do estresse em catetos (Tayassu tajacu) submetidos à captura e contenção em diferentes horários da manhã em Mossoró, RN. Ciênc. Anim. Bras. 9(1):170-176.

Bloor B.C. \& Flacke W.E. 1982. Reduction in halothane anesthetic requirement by clonidine, an alpha-adrenergic agonist. Anesth. Analg. 61(9): 741-745.

Costa D.S., Henry M. \& Paula T.A.R. 2004. Espermatogênese de Catetos (Tayassu tajacu). Arq. Bras. Med. Vet. Zootec. 56(1):46-51.

Dahl G.T., Woldseth B. \& Lindemann R. 2012. Metabolic acidosis mimicking diabetic ketoacidosis after use of calorie-free mineral water. Eur. J. Pediatr. 171(9):1405-1407.

Doi M. \& Ikeda K. 1978. Respiratory effects of sevoflurane. Anesth. Analg. 66(1):241-244.

Eger E.I. 2001. Age, minimum alveolar concentration, and minimum alveolar anesthetic concentration-awake. Anesth. Analg. 93(4):947953.

Eger E.I., Saidman L.J. \& Brandstater B.M. 1965. Minimum alveolar anesthetic concentration: A standard of anesthetic potency. Anesthesiology 26(6):756-763.

Egger C., Mccrackin M.A., Hofmeister E., Touzot-Jourde G. \& Rohrbach B. 2009. Efficacy of preanesthetic intramuscular administration of ephedrine for prevention of anesthesia-induced hypotension in cats and dogs. Can. Vet. J. 50(2):179-84.

Fahlman A. 2005. Anaesthesia of wild carnivores and primates. MSc. Dissertation, Department of Clinical Sciences, Faculty of Veterinary Medicine and Animal Science, Swedish University of Agricultural Sciences, Uppsala, Sweden. 36p.

Fantoni D.T., Marchoini G.G., Ida K.K., Belo J.N.B., Zoppa A.L.V., Silva L.C.L.C. \& Ambrósio A.M. 2013. Effect of ephedrine and phenylephrine on cardiopulmonary parameters in horses undergoing elective surgery. Vet. Anaesth. Analg. 40(4):367-74.

Farias D.C., Vasconcelos L.P.S., Turbino N.R., Monzem S., Luiz M.A., Paz R.C. \& Guimarães L.D. 2009. Técnicas de contenção química em cachorro-do-mato (Cerdocyon thous). Acta Sci. Vet. 37(3):265-269.

Galloway D.S., Ko J.C.H., Mandsage R.E., Reaugh H.V., Inoue T., Payton M.E. \& Portillo E. 2004. Anesthetic indices of sevoflurane and isoflurane in unpremedicated dogs. J. Am. Vet. Med. Assoc. 225(5):700-704.

Golden A.L., Bright J.M., Daniel G.B., Fefee D., Schmidt D. \& Harvey R.C. 1998. Cardiovascular effects of $\alpha$-2adrenergic receptor agonist medetomidine in clinically normal cats anesthetized with isoflurane. Am. J. Vet. Res. 59(4):509-513.

Greene S.A., Benson G.J., Tranquilli W.J. \& Grimm K.A. 2004. Effect of isoflurane, atracurium, fentanyl, and noxious stimulation on bispectral index in pigs. Comp. Med. 54(4):397-403.

Haga H.A., Ranheim B. \& Spadavecchia C. 2011. Effects of isoflurane upon minimum alveolar concentration and cerebral cortex depression in pigs and goats: an interspecies comparison. Vet. Journal 187(2):217-220.

Haskins S.C. 2013. Monitoração de paciente anestesiado, p.582-611 In: Thurmon J.C., Tranquilli W.J. \& Grimm K.A. (Eds), Lumb and Jones' Anestesiologia e Analgesia Veterinária. 4를. ed. Roca, São Paulo.

Hellyer P.W., Dodam J.R. \& Light G.S. 1991. Dynamic baroreflex sensitivity in anesthetized horses, maintained at 1.25 to 1.3 minimal alveolar concentration of halothane. Am. J. Vet. Res. 52(10):1672-1675.

Kimme P., Ledin T. \& Sjöberg F. 2007. Dose effect of sevoflurane and isoflurane anesthetics on cortical blood flow during controlled hypotension in the pig. Acta. Anaesthesiol. Scand.51(5):607-613.
Kurita T., Takata K., Uraoka M., Morita K., Sanjo Y., Katoh T. \& Sato S. 2007. The influence of hemorrhagic shock on the minimum alveolar anesthetic concentration of isoflurane in a swine model. Anesth. Analg. 105(6):1639-1643.

Langley M.S. \& Heel R.C. 1988. Propofol: a review of its pharmacodynamics and pharmacokinetic properties and use as an intravenous anaesthetic. Drugs 35(4):334-372.

Lukasik M.V., 1999. Premedication and sedation, p.71-86. In: Seymour C. \& Gleed R. (Eds), Manual of Small Animal Anesthesia and Analgesia. British Small Animal Veterinary Association, Shurdington.

Lundeen G., Manohar M. \& Parks C. 1983. Systemic distribution of blood flow in swine while awake and during 1.0 and 1.5 MAC isoflurane anesthesia with or without 50\% nitrous oxide. Anesth. Analg. 62(5):499-512.

Muir W.W. \& Morais H.S.A. 2013. Fisiologia do equilíbrio ácido-base, p.190-206. In: Thurmon J.C., Tranquilli W.J. \& Grimm K.A. (Eds), Lumb and Jones' Anestesiologia e Analgesia Veterinária. 4ª ed. Roca, São Paulo.

Oliva V.N.L.S. \& Fantoni D.T. 2009. Anestesia inalatória, p.174-183. In: Fantoni D.T. \& Cortopoassi R.S.G. (Eds), Anestesia em Cães e Gatos. 2 $2^{\mathrm{a}}$ ed. Roca, São Paulo.

Oliveira C.R.D. \& Elias L. 2006. Hipnóticos não-barbitúricos, p.291-305. In: Cangiani L.M., Posso I.P., Potério G.M.B. \& Nogueira C.S. (Eds), Tratado de Anestesiologia SAESP. 6 ${ }^{\mathrm{a}}$ ed. Atheneu, São Paulo.

Ortolani E.L. 2003. Diagnóstico e tratamento de alterações ácidos-básicas em ruminantes, p.17-29. In: González FH.D. \& Campos R. (Eds), Anais do I Simpósio de Patologia Clínica Veterinária da Região Sul do Brasil, Porto Alegre, RS. (Resumo)

Pulz R.S. 2006. Anestesia inalatória e anestesia balanceada em cirurgia torácica experimental em suínos. Tese de Doutorado em ciências veterinárias na área de cirurgia experimental, Universidade Federal do Rio Grande do Sul, Porto Alegre, RS. 88p.

Quasha A.L., Eger E.I. \& Tinker J.H. 1980. Determinations and Applications of MAC. Anesthesiology 53(4):315-334.

Raisis A.L., Young L.E., Blissitt K.J., Brearley J.C., Meire H.B., Taylor P.M. \& Lekeux P. 2000. A comparison of the haemodynamic effects of isoflurane and halothane anaesthesia in horses. Equine Vet. J. 32(4):318-326.

Souza A.L.P., Paula V.V., Cavalcante P.H. \& Oliveira M.F. 2008. Efeito da pré-medicação com acepromazina ou xilazina na indução da anestesia dissociativa com cetamina e diazepam em catetos (Tayassu tajacu). Ciênc. Anim. Bras. 9(4):1114-1120.

Souza A.L.P., Castelo T.S., Queiroz J.P.A.F., Barros I.O., Paula V.V., Oliveira M.F. \& Silva A.R. 2009. Evaluation of anesthetic protocol for the collection of semen from captive collared peccaries (Tayassu tajacu) by electroejaculation. Anim. Reprod. Sci. 116(3/4):370-375.

Steffey E.P. \& Howland D.J.R. 1978. Potency of enflurane in dogs: comparison with halothane and isoflurane. Am. J. Vet. Res. 39(4):573-577.

Steffey E.P. \& Mama K.R. 2013. Anestésicos inalatórios, p.385-417. In: Thurmon J.C., Tranquilli W.J. \& Grimm K.A. (Eds), Lumb and Jones' Anestesiologia e Analgesia Veterinária. 4aㅡ ed. Roca, São Paulo.

Sutherland-Smith M., Campos J.M., Cramer C., Thorstadt C., Toone W. \& Morris P.J. 2004. Immobilization of chacoan peccaries (Catagonus wagneri) using medetomidine, telazol, and ketamine. J. Wildl. Dis. 40(4): 731-736.

Valverde A., Morey T.E. \& Hernandez J. 2003. Validation of several types of noxious stimuli for use in determining the minimum alveolar concentration for inhalation anesthetics in dogs and rabbits. Am. J. Vet. Res. 64(8):957-962.

Wilson D.V., Evans A.T., Carpenter R.A. \& Mullineaux D.R. 2004. The effect of four anesthetic protocols on splenic size in dogs. Vet. Anaesth. Analg. 31(2):102-108. 